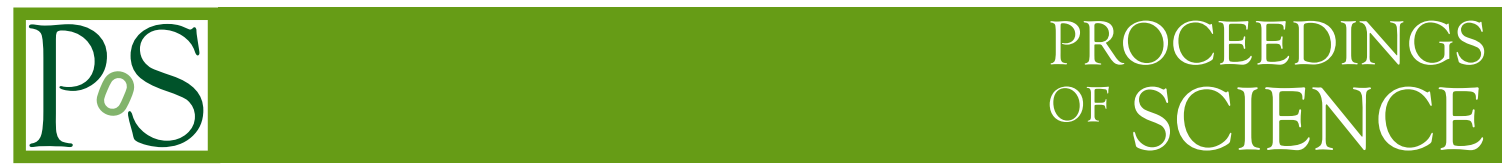

\title{
Bogomolny Equations from the Pseudoanalytic Functions Viewpoint
}

\section{Tinatin Supatashvili*}

Tbilisi State University,

1 Ilia Chavchavadze Avenue, Tbilisi, Georgia

E-mail: tinatin.supatashvili969@ens.tsu.edu.ge

In the following paper we are going to study a special kind of solitons, called vortices. We will define the topological number for them, called the vortex number. Switching to complex variables and using a different approach from the one used by Jaffe and Taubes in the famous monograph "Vortices and monopoles", 1980, in particular, using the theorems known in pseudoanalytic function theory, we construct the vortex solutions of the field equations for our problem and write out the vortex numbers for them that will be characterized by the zeroes of each possible solution.

RDP online workshop "Recent Advances in Mathematical Physics" - Regio2020,

5-6 December 2020

online

${ }^{*}$ Speaker 


\section{Introduction}

In quantum field theory there is a class of solutions of field equations called "solitons". They are solutions of nonlinear field equations and have finite energy. They can be identified as lumps of energy that are localized in some finite area of space. We can consider either static or timedependent solutions. A so-called topological number can be assigned to them to characterize and distinguish them from each other. The systems possessing this type of solutions are realizable in condensed matter physics, though there is no evidence of them being associated with point-like particles.

When we have a couple of solitons, they may interact with each other: either repel or attract one another. The nature of the interaction depends on their respective topological numbers and the parameters of the system. Some of the examples of solitons are objects like: strings, vortices, instantons. The simplest soliton appears in the case of a single real scalar field in $(1+1)$-dimensional space-time with Higgs potential. Other than the classical vacuum solution there exists a non-trivial solution called kink. Each kink carries its own topological number that is determined, as in the general case, from the mapping of the configuration space to the field space.

In systems possessing local symmetry vortex is the simplest soliton. It can appear as a solution in complex scalar field theory with $U(1)$ local symmetry, including Higgs potential in $(2+1)$-dimensional space-time. In the cosmological context they can be created during the phase transitions that occurred after the Big Bang; in this context they are called cosmic strings. The fact that they have not been discovered can be accounted for by the inflation era.

One of the interesting solitons is the 't Hooft-Polyakov monopole (1974). Here the existence of soliton-type solutions, i.e., monopoles, is related to the topology of the system, in particular, the second homotopy group of the quotient space ${ }^{G} / H$ (viewed as a smooth manifold), $\pi_{2}\left({ }^{G} / H\right.$ ), where $\mathrm{G}$ is a local gauge group and $\mathrm{H}$ is the subgroup of $\mathrm{G}$ which leaves the field space of solutions invariant.

The possibility of the theoretical existence of solitons can be seen from the scale arguments (Derrick 1964). Here we see the importance of the dimension and the symmetry of the system under consideration as well as whether there is a Higgs potential or not. For example, in case a system does not possess the local symmetry, there are no solitons in $(d+1)$-dimensional space-time with $d>2$. They are also absent in purely gauge theories for $d \neq 4$.

\section{Physical picture}

Consider the following Lagrangian for a complex scalar field $\varphi(x)$ in $(2+1)$ - dimensional space-time with U(1) gauge symmetry and Higgs potential [4]:

$$
\mathcal{L}=-\frac{1}{4} F_{\mu \nu} F^{\mu \nu}+\left(D_{\mu} \varphi\right) \overline{\left(D^{\mu} \varphi\right)}-V(\varphi),
$$

where $\mu, v=0,1,2 . \overline{\left(D^{\mu} \varphi\right)}-$ denotes complex conjugation and summation is considered via the repeated indices with the Minkowski metric tensor $\eta_{\mu \nu}=(1,-1,-1)$. Here $D_{\mu}=\partial_{\mu}-i e A_{\mu}, \partial_{\mu} \equiv$ $\frac{\partial}{\partial x^{\mu}}$, is a covariant derivative and $e=$ const. 


$$
\begin{gathered}
F_{\mu \nu}=\partial_{\mu} A_{\nu}-\partial_{\nu} A_{\mu}, \\
V(\varphi)=\frac{\lambda}{2}\left(|\varphi|^{2}-v\right)^{2}
\end{gathered}
$$

are the field tensor and Higgs potential, respectively, $v$ and $\lambda$ are real parameters.

The local $U(1)$ transformation for scalar field and gauge potential has the following form:

$$
\begin{gathered}
\varphi(x) \longrightarrow e^{i \alpha(x)} \varphi(x), \\
A_{\mu}(x) \longrightarrow A_{\mu}(x)+\frac{1}{e} \partial_{\mu} \alpha(x) .
\end{gathered}
$$

We are interested only in static configurations, that is $\varphi=\varphi(\mathbf{x}), A_{i}=A_{i}(\mathbf{x}), A_{0}=0$, where $i=1,2$ and $\varphi(\mathbf{x})=\varphi\left(x_{1}, x_{2}\right)$, then for the energy functional we have

$$
E\left[A_{i}, \varphi\right]=\int_{\mathbb{R}^{2}} d^{2} x\left[\frac{1}{4} F_{i j} F^{i j}+\left(D_{i} \varphi\right) \overline{\left(D^{i} \varphi\right)}+\frac{\lambda}{2}\left(|\varphi|^{2}-v\right)^{2}\right] .
$$

For finiteness of the energy we require

$$
\left\{\begin{array}{l}
|\varphi| \longrightarrow v, \\
\left(D_{i} \varphi\right) \longrightarrow 0
\end{array}\right.
$$

when $|\mathbf{x}| \rightarrow \infty$.

\section{Formulation of problem}

Looking at the same problem from the differential geometrical viewpoint, since solutions are time independent, we will need two bundles with the base space $\mathbb{R}^{2}$ :

1. Principal bundle with the fiber $G=U(1)$,

2. vector bundle associated with this principal bundle.

In our case, we have $\mathbb{C}$ as a fiber and $G$ group acts on it by the simple multiplication of two complex numbers.

From now on, we will look at the complex field $\varphi(x)=\varphi_{1}(x)+i \varphi_{2}(x)$ as the section of Hermitian line bundle over the $\mathbb{R}^{2} . A_{i}(\mathbf{x})$ - now are components of the connection on $\mathbb{R}^{2}$. In the coordinates $\left(x_{1}, x_{2}\right)$ the connection will be

$$
A(\mathbf{x})=A_{1}(\mathbf{x}) d x_{1}+A_{2}(\mathbf{x}) d x_{2} .
$$

$F_{i j}$ are components of the curvature associated with this connection. It is known that in general

$$
F=d A+A \wedge A=\frac{1}{2} \sum_{i, j=1}^{2} F_{i j} d x_{i} \wedge d x_{j},
$$

where $d: \Omega^{k}\left(\mathbb{R}^{2}\right) \rightarrow \Omega^{k+1}\left(\mathbb{R}^{2}\right)$ is exterior derivative of the smooth $k$-forms $\Omega^{k}\left(\mathbb{R}^{2}\right)$ and $\wedge$ is a wedge product between the differential forms. In our case the second term in (3) vanishes and we are left with 


$$
F=d A
$$

so

$$
F_{i j}=\partial_{i} A_{j}-\partial_{j} A_{i},
$$

as we had for the Field tensor. To summarize the objects we have in the language of differential forms:

$$
\begin{gathered}
\varphi-\mathbb{C} \text {-valued } 0 \text {-form; } \\
A-\mathfrak{u}(1) \text {-algebra valued } 1 \text {-form; }
\end{gathered}
$$$$
F-\mathfrak{u}(1) \text {-algebra valued } 2-\text { form. }
$$

Using these and constructing so called "Yang-Mills-Higgs" action with $U(1)$ local symmetry, we arrive to

$$
\mathcal{A}\left[A_{i}, \varphi\right]=\frac{1}{2} \int_{\mathbb{R}^{2}}\left[F_{A} \wedge * F_{A}+\left(D_{A} \varphi\right) \wedge * \overline{\left(D_{A} \varphi\right)}+\frac{\lambda}{2} *\left(|\varphi|^{2}-1\right)^{2}\right],
$$

which is an analogue of the energy functional (2). Here " $*$ " is a Hodge star operator $*: \Omega^{k}\left(\mathbb{R}^{2}\right) \rightarrow$ $\Omega^{\operatorname{dim}\left(\mathbb{R}^{2}\right)-k}\left(\mathbb{R}^{2}\right)$ and

$$
D_{A} \varphi=\left(\nabla_{A}\right)_{1} \varphi d x_{1}+\left(\nabla_{A}\right)_{2} \varphi d x_{2},
$$

where $\left(\nabla_{A}\right)_{i} \varphi$ is a covariant derivative of $\varphi$ and gives us again a section in the following way

$$
\left(\nabla_{A}\right)_{i} \varphi=\nabla_{i} \varphi+\rho\left(A_{i}\right) \varphi
$$

Here $\nabla_{i} \equiv \partial_{i}$ and $\rho\left(A_{i}\right)$ is a representation of the Lie algebra corresponding to the considered group. The representation space is chosen to be a fiber of the associated vector bundle. In our case from (5) follows that $\left(\nabla_{A}\right)_{i} \varphi=\left(\partial_{i}-i A\right) \varphi$.

Let us now define the vortex number

$$
N=\frac{1}{2 \pi} \int_{\mathbb{R}^{2}} F, \quad N \in \mathbb{Z}
$$

For the field equations we will take a variance of (4) with respect to $\bar{\varphi}$ and $A$ and get:

$$
d * F_{A}=\frac{i}{2} *\left(\varphi \overline{D_{A} \varphi}-\bar{\varphi} D_{A} \varphi\right), \quad D_{A} * D_{A} \varphi=\frac{\lambda}{2} *\left(|\varphi|^{2}-1\right) \varphi,
$$

or in components

$$
\begin{gathered}
\partial_{i} F_{i j}=-\frac{i}{2}\left(\varphi\left(\partial_{j}+i A_{j}\right) \bar{\varphi}-\bar{\varphi}\left(\partial_{j}-i A_{j}\right) \varphi\right)=\operatorname{Im}\left(\varphi \overline{\left(\nabla_{A}\right)_{j} \varphi}\right) \equiv J_{j}, \\
\nabla_{A}^{2} \varphi=\frac{\lambda}{2} \varphi\left(|\varphi|^{2}-1\right) .
\end{gathered}
$$

It is clear that they coincide with the field equations for the stationary case, when the Lagrangian has (1) form.

Taking the special case $\lambda=1$, we can rewrite (4) as 


$$
\begin{aligned}
\mathcal{A}\left[A_{i}, \varphi\right]=\int_{\mathbb{R}^{2}} d^{2} x & \left\{\frac{1}{2}\left[\left(\partial_{1} \varphi_{1}+A_{1} \varphi_{2}\right) \mp\left(\partial_{2} \varphi_{2}-A_{2} \varphi_{1}\right)\right]^{2}+\right. \\
& +\frac{1}{2}\left[\left(\partial_{2} \varphi_{1}+A_{2} \varphi_{2}\right) \pm\left(\partial_{1} \varphi_{2}-A_{1} \varphi_{1}\right)\right]^{2}+ \\
& \left.+\frac{1}{2}\left[F_{12} \pm \frac{1}{2}\left(\varphi_{1}^{2}+\varphi_{2}^{2}-1\right)\right]^{2}\right\} \pm \frac{1}{2} \int_{\mathbb{R}^{2}} d^{2} x F_{12} .
\end{aligned}
$$

From (9) follows that $\mathcal{A} \geq \pi|N|$, where $N$ is defined by (6). If $N>0$, minimum of the action is achieved when

$$
\begin{gathered}
\left(\partial_{1} \varphi_{1}+A_{1} \varphi_{2}\right)-\left(\partial_{2} \varphi_{2}-A_{2} \varphi_{1}\right)=0 \\
\left(\partial_{2} \varphi_{1}+A_{2} \varphi_{2}\right)+\left(\partial_{1} \varphi_{2}-A_{1} \varphi_{1}\right)=0, \\
F_{12}+\frac{1}{2}\left(\varphi_{1}^{2}+\varphi_{2}^{2}-1\right)=0 .
\end{gathered}
$$

Solutions of these equations also satisfy the equations (7), (8). For $N<0$, similar equations are obtained.

\section{Complex variables}

Next step is to go to the complex variables via

$$
z=x_{1}+i x_{2}, \quad \bar{z}=x_{1}-i x_{2}, \quad \frac{\partial}{\partial z} \equiv \partial_{z}=\frac{1}{2}\left(\partial_{1}-i \partial_{2}\right), \quad \frac{\partial}{\partial \bar{z}} \equiv \partial_{\bar{z}}=\frac{1}{2}\left(\partial_{1}+i \partial_{2}\right) .
$$

Decomposing $\varphi$ into its real and imaginary parts and writing $A$ in the new coordinates $(z, \bar{z})$

$$
\varphi=\varphi_{1}+i \varphi_{2}, \quad A=\alpha d z+\bar{\alpha} d \bar{z}
$$

where $\alpha=\frac{1}{2}\left(A_{1}-i A_{2}\right), \bar{\alpha}=\frac{1}{2}\left(A_{1}+i A_{2}\right)$.

The equations (10), (11), (12) in complex variables will become

$$
\begin{gathered}
\left(\partial_{z}+i \alpha\right) \bar{\varphi}+\left(\partial_{\bar{z}}-i \bar{\alpha}\right) \varphi=0, \\
\left(\partial_{z}+i \alpha\right) \bar{\varphi}-\left(\partial_{\bar{z}}-i \bar{\alpha}\right) \varphi=0, \\
2 i\left(\partial_{\bar{z}} \alpha-\partial_{z} \bar{\alpha}\right)=4 \operatorname{Im}\left(\partial_{\bar{z}} \alpha\right)=\frac{1}{2}(1-\varphi \bar{\varphi}) .
\end{gathered}
$$

It is clear that (13) and (14) are real and imaginary parts of the expression

$$
D_{A} \varphi-i * D_{A} \varphi=2\left(\partial_{\bar{z}}-i \bar{\alpha}\right) \varphi d \bar{z}=0,
$$

from which follows that

$$
\left(\partial_{\bar{z}}-i \bar{\alpha}\right) \varphi=0 .
$$

This is the main equation that we are going to study in this paper. 
Note that

$$
\begin{aligned}
D_{A} \varphi & =\left(\partial-i A_{1}\right) \varphi d x_{1}+\left(\partial-i A_{2}\right) \varphi d x_{2} \\
& =\frac{1}{2}\left[\left(\partial_{z}+\partial_{\bar{z}}\right)-i(\alpha+\bar{\alpha})\right] \varphi(d z+d \bar{z})+\frac{1}{2 i}\left[i\left(\partial_{z}-\partial_{\bar{z}}\right)+i(\alpha-\bar{\alpha})\right] \varphi(d z-d \bar{z}) \\
& =\left(\partial_{z}-i \alpha\right) \varphi d z+\left(\partial_{\bar{z}}-i \bar{\alpha}\right) \varphi d \bar{z}
\end{aligned}
$$

Using that $* d x_{1}=d x_{2} ; * d x_{2}=-d x_{1} \rightarrow * d z=-i d z$ and $* d \bar{z}=i d \bar{z}$, we obtain the variational equations in complex variables

$$
\begin{aligned}
D_{A} * D_{A} \varphi & =\left[\left(\partial_{z}-i \alpha\right) \varphi d z+\left(\partial_{\bar{z}}-i \bar{\alpha}\right) \varphi d \bar{z}\right]\left[\left(-\partial_{z}-i \alpha\right) \varphi d z+\left(\partial_{\bar{z}}+i \bar{\alpha}\right) \varphi d \bar{z}\right] \\
& =\left(2 i\left(\partial_{z} \partial_{\bar{z}}-\alpha \bar{\alpha}\right) \varphi+\partial_{z}(\bar{\alpha} \varphi)+\partial_{\bar{z}}(\alpha \varphi)+\alpha \partial_{\bar{z}} \varphi+\bar{\alpha} \partial_{z} \varphi\right) d z \wedge d \bar{z}, \\
\frac{\lambda}{2} *\left(|\varphi|^{2}\right. & -1) \varphi=\left(\frac{\lambda}{2}\left(|\varphi|^{2}-1\right) \varphi\right) d x_{1} \wedge d x_{2}=\left(\frac{\lambda}{2}\left(|\varphi|^{2}-1\right) \varphi\right) \frac{i}{2} d z \wedge d \bar{z},
\end{aligned}
$$

so $D_{A} * D_{A} \varphi=\frac{\lambda}{2} *\left(|\varphi|^{2}-1\right) \varphi$ and $d * F_{A}=\frac{i}{2} *\left(\varphi \overline{D_{A} \varphi}-\bar{\varphi} D_{A} \varphi\right)$ in components will be:

$$
4\left(\partial_{z} \partial_{\bar{z}}-\alpha \bar{\alpha}\right) \varphi-2 i\left(\partial_{z} \bar{\alpha}+\partial_{\bar{z}} \alpha+\alpha \partial_{\bar{z}}+\bar{\alpha} \partial_{z}\right) \varphi=\frac{\lambda}{2}\left(|\varphi|^{2}-1\right) \varphi
$$

and

$$
\begin{aligned}
& \left(\partial_{z}-\partial_{\bar{z}}\right) \operatorname{Im}\left(\partial_{\bar{z}} \alpha\right)=\frac{1}{4 i} \operatorname{Im}\left(\varphi\left(\partial_{z}+\partial_{\bar{z}}\right) \bar{\varphi}\right)-\frac{1}{8}(\alpha+\bar{\alpha})|\varphi|^{2}, \\
& \left(\partial_{z}+\partial_{\bar{z}}\right) \operatorname{Im}\left(\partial_{\bar{z}} \alpha\right)=\frac{1}{4 i} \operatorname{Im}\left(\varphi\left(\partial_{z}-\partial_{\bar{z}}\right) \bar{\varphi}\right)-\frac{1}{8}(\alpha-\bar{\alpha})|\varphi|^{2}
\end{aligned}
$$

respectively.

In the next section we will analyze the following theorem from [4] in spirit of the paper [1] using the theory of the pseudoanalytic functions [3], [5].

Theorem 1. Given an integer $N \geq 0$ and a set $\left\{z_{i}\right\}, i=1, \ldots, N$, of $N$ points in $\mathbb{C}$, there exists a finite action solution to equations (10), (11), (12), unique up to gauge equivalence, with the following properties:

1. The solution is globally smooth.

2. The zeros of $\varphi$ are the set of point $\left\{z_{i}\right\}$, and

$$
\varphi(z, \bar{z}) \sim c_{i}\left(z-z_{i}\right)^{n_{i}}, c_{i} \neq 0,
$$

as $z \rightarrow z_{i} . n_{i}$ is multiplicity of $z_{i}$ in the set $\left\{z_{i}\right\}$.

3.

$$
N=\frac{1}{2 \pi} \int_{\mathbb{R}^{2}} F_{A}=\sum_{\text {distinct } z_{i}} n_{i}=\frac{1}{\pi} \mathcal{A}
$$




\section{On pseudoanalytic functions}

Pseudoanalytic functions are a class of complex-valued functions that obey the generalized Cauchy-Riemann equations [3], [5]. In this section we give some concepts and basic facts from the theory of the pseudoanalytic functions.

Let $\Omega$ be a simply connected domain in $\mathbb{R}^{2}$. A pair of complex functions $F$ and $G$ in the domain $\Omega$, which have Hölder continuous partial derivatives with respect to the real variables, is called a generating pair, if

$$
\operatorname{Im}(\bar{F} G)>0 \quad \text { in } \Omega
$$

inequality holds.

Let $\omega: \Omega \rightarrow \mathbf{C}$ be a complex valued function in the domain $\Omega \subset \mathbf{C}$. It is clear that there exist real-valued functions $\phi(z)$ and $\psi(z)$ such that

$$
\omega(z)=\phi(z) F(z)+\psi(z) G(z) .
$$

We say that $\omega$ has $(F, G)$-derivative, denoted by $\dot{\omega}\left(z_{0}\right)$, at $z_{0} \in \Omega$ if the limit

$$
\dot{\omega}\left(z_{0}\right)=\lim _{z \rightarrow z_{0}} \frac{\omega(z)-\phi\left(z_{0}\right) F(z)-\psi\left(z_{0}\right) G(z)}{z-z_{0}}
$$

exists and it is finite.

We will call $\omega(F, G)$-pseudoanalytic in the domain $\Omega$ if $\dot{\omega}$ exists everywhere in $\Omega$. Denote by $A_{\Omega}(F, G)$ the space of $(F, G)$-pseudoanalytic functions. This space contains the space $H(\Omega)$ of analytic functions and main properties of analytic functions extend on $A_{\Omega}(F, G)$. Namely, the following important theorem holds.

Theorem 2. A function $\omega$ is $(F, G)$-pseudoanalytic in domain $\Omega$ if and only if $\omega$ satisfies the following generalized nonhomogenous Cauchy-Riemann equation (so called Carleman-Bers-Vekua equation, see [2])

$$
\omega_{\bar{z}}=a \omega+b \bar{\omega}
$$

where

$$
a=-\frac{\bar{F} G_{\bar{z}}-F_{\bar{z}} \bar{G}}{F \bar{G}-\bar{F} G}, \quad b=\frac{F G_{\bar{z}}-F_{\bar{z}} G}{F \bar{G}-\bar{F} G}
$$

are defined on the domain $\Omega$ and satisfy the regularity conditions (see [2]) $a, b \in L_{p, 2}(\mathbb{C})$. $\omega_{\bar{z}} \equiv \partial_{\bar{z}} \omega$ is generalized derivative in the Sobolev sense with respect to $\bar{z}$.

The solutions of the equations are elements of the space $A_{\Omega}(F, G)$ and the following, so called similarity principle, is valid:

$$
\omega=\Phi e^{s}
$$

where $\Phi(z)$ is analytic and

$$
s=\left\{\begin{array}{l}
T_{\mathbb{C}}\left[a+b \frac{\bar{\omega}}{\omega}\right], \text { if } \omega(z) \neq 0 \quad z \in \Omega, \\
T_{\mathbb{C}}[a+b], \text { if } \omega(z)=0 \quad z \in \Omega .
\end{array}\right.
$$


The integral

$$
q(z)=T_{\mathbb{C}}[g(z)]=-\frac{1}{\pi} \int_{\mathbb{C}} \frac{g(\lambda)}{\lambda-z} d \xi d \eta, \quad \lambda=\xi+i \eta
$$

for $g(z) \in L_{p, 2}(\mathbb{C}), p>2$ is well defined, $q(z) \in D_{\bar{z}} \mathbb{C}$ and $\frac{\partial T_{E}[g(z)]}{\partial \bar{z}}=g(z)$. Here $D_{\bar{z}} \mathbb{C}$ is a linear space of functions that have generalized derivative in the Sobolev sense with respect to $\bar{z}$.

From the representation (19) follows the following important property of pseudoanalytic functions.

Corollary 1. (Carleman's theorem) A pseudoanalytic function, which does not vanish identically, has only isolated zeros.

Returning to the equation $\varphi_{\bar{z}}=i \bar{\alpha} \varphi$ and comparing it to (18), we conclude that $b=0$ and $a=i \bar{\alpha}$. We will look at $\varphi$ as the pseudoanalytic function and use the representation (19) to get

$$
\begin{aligned}
\varphi(z)=\Phi e^{T[a]} & =\Phi(z) \exp \left\{-\frac{1}{\pi} \int_{\mathbb{C}} \frac{a(\lambda)}{\lambda-z} d \xi d \eta\right\} \\
& =\Phi(z) \exp \left\{-\frac{i}{\pi} \int_{\mathbb{C}} \frac{\bar{\alpha}(\lambda)}{\lambda-z} d \xi d \eta\right\} \\
& =\Phi(z) \exp \left\{-\frac{i}{2 \pi} \int_{\mathbb{C}} \frac{\left(A_{1}+i A_{2}\right)(\lambda)}{\lambda-z} d \xi d \eta\right\} \\
& =\Phi(z) \exp \left\{\frac{1}{4 \pi} \int_{\mathbb{C}} \frac{\left(A_{1}+i A_{2}\right)(\lambda)}{\lambda-z} d \lambda d \bar{\lambda}\right\} .
\end{aligned}
$$

The same result is obtained by using the $\bar{\partial}$-Poincaré lemma [4].

For it to be a solution of our problem, it needs to satisfy the following condition:

$$
|\varphi(z)|=\left|\Phi(z) \exp \left\{\frac{1}{4 \pi} \int_{\mathbb{C}} \frac{\left(A_{1}+i A_{2}\right)(\lambda)}{\lambda-z} d \lambda d \bar{\lambda}\right\}\right| \rightarrow 1 \text { as }|z| \rightarrow \infty .
$$

Now using the Corollary 2.1, we arrive to the statement: if $\varphi$ has zeros, they are isolated.

For asymptotic behavior near zeros of $\varphi(z)$, we use the same arguments as in [4]. Namely, suppose $\varphi\left(z_{k}\right)=0, z_{k} \in \Omega$. according to our representation of $\varphi, \Phi\left(z_{k}\right)=0$ and since $\Phi(z)$ is analytic, in the neighborhood of $z_{k}$ we have

$$
\Phi(z)=g(z)\left(z-z_{k}\right)^{n_{k}},
$$

where $n_{k}$ is multiplicity of $z_{k}, g(z)$ is analytic function and $g\left(z_{k}\right) \neq 0$. Furthermore, for $\varphi$ in the neighborhood of $z_{k}$ we can write:

$$
\varphi=g(z)\left(z-z_{k}\right)^{n_{k}} e^{s}=h(z)\left(z-z_{k}\right)^{n_{k}},
$$

where $h(z)=g(z) e^{s}$ and $h\left(z_{k}\right) \neq 0$. We can rewrite the previous expression as

$$
\varphi(z)=|h(z)| e^{i \arg (h(z))}\left|z-z_{k}\right|^{n_{k}} e^{i n_{k} \arg \left(z-z_{k}\right)} \equiv e^{u+i \theta} .
$$

From it we see that all zeros of $\varphi(z)$ are at the same time singular points for the "phase", i.e. $\theta(z)=\arg (h(z))+n_{k} \arg \left(z-z_{k}\right)$ and the singularity comes from the second term, since $h(z)$ does not vanish at $z_{k}$. 


\section{Winding and vortex numbers}

Let us construct a map $f: S^{1} \rightarrow S^{1}$, where $S^{1}$ is the unit circle in $\mathbb{R}^{2} \cong \mathbb{C}$, and $S^{\prime 1}$ is a set of values of $\varphi(z)$, when $|z| \rightarrow \infty$. For this consider the function

$$
f(z)=e^{i \beta(z)}
$$

and its restriction

$$
\beta(z)=\beta(|z|, \arg (z)+2 \pi)=\beta(|z|, \arg (z))+2 \pi \operatorname{deg}(f) .
$$

The degree of this map, as well as the winding number is

$$
\operatorname{deg}(f)=\text { Winding number }=\frac{1}{2 \pi i} \oint_{S^{1}} \frac{\partial f}{f} d z .
$$

Construct $f$ from $\varphi$ in the following way

$$
f=\lim _{|z| \rightarrow \infty} \varphi(z)
$$

so $S^{\prime 1}$ is a circle with radius 1 in the space of the values of $\varphi$. Using (21), we can represent $f$ of the form

$$
f=\exp \left\{2 i \sum_{k} \arg \left(z-z_{k}\right)\right\} .
$$

Putting this into (22) we obtain

$$
\operatorname{deg}(f)=\frac{1}{2 \pi i} \oint_{S^{1}} \frac{\partial f}{f} d z=\frac{1}{2 \pi i} \oint_{S^{1}} \frac{1}{z-z_{k}} d z=\sum_{\text {distinct } z_{i}} n_{i},
$$

so

$$
\operatorname{deg}(f)=\text { Winding number }=\sum_{\text {distinct } z_{i}} n_{i} .
$$

Now, using the asymptotic behavior, we can compute components of the connection in the coordinates $(z, \bar{z})$. In particular, when $|z| \rightarrow \infty,|\varphi| \rightarrow 1$ and $D_{A} \varphi=(d \varphi-i A \varphi) \rightarrow 0$, that means $d \varphi \rightarrow i A \varphi$. On and beyond a sufficiently large circle $|\varphi|=1, d \varphi=i A \varphi$. Writing the latter one in components

$$
\partial_{z} \varphi d z+\partial_{\bar{z}} \varphi d \bar{z}=i \alpha \varphi d z+i \bar{\alpha} \varphi d \bar{z}
$$

From this we obtain two equations

$$
\left\{\begin{array}{l}
\left(\partial_{\bar{z}}-i \bar{\alpha}\right) \varphi=0 \\
\left(\partial_{z}-i \alpha\right) \varphi=0
\end{array}\right.
$$

The first one is the same as the equation (16) that we were studying. With the use of (23) for the $\varphi$, when $|z| \rightarrow \infty$, let us solve the second equation 


$$
\left(\partial_{z}-i \alpha\right) \exp \left\{2 i \sum_{k} \arg \left(z-z_{k}\right)\right\}=0
$$

of the system (24) with respect $\alpha$ :

$$
\alpha(z)=\partial_{z} 2 \sum_{k} \arg \left(z-z_{k}\right)=-i \sum_{k} \frac{\bar{z}-\overline{z_{k}}}{\left|z-z_{k}\right|^{2}}=-i \sum_{k} \frac{1}{z-z_{k}},
$$

here $z \in S^{1}$ with sufficiently large radius. For any $z \in \mathbb{C}$ we have the representation of $\alpha$

$$
\alpha(z)=-i \chi(z, \bar{z}) \sum_{k} \frac{1}{z-z_{k}},
$$

where $\chi(z, \bar{z})$ is an arbitrary function with the property $\lim _{|z| \rightarrow \infty} \chi(z, \bar{z})=1$ and now compute the vortex number:

$$
\begin{aligned}
N & =\frac{1}{2 \pi} \int_{\mathbb{R}^{2}} F_{A}=\frac{1}{2 \pi} \oint_{S^{1}(|z| \rightarrow \infty)} A=\frac{1}{2 \pi}\left(-i \oint_{S^{1}} \sum_{k} \frac{1}{z-z_{k}} d z+i \oint_{S^{1}} \sum_{k} \frac{1}{\bar{z}-\bar{z}_{k}} d \bar{z}\right)= \\
& =\sum_{\text {distinct } z_{i}} n_{i} .
\end{aligned}
$$

Therefore, we have obtained another proof of the Theorem 1.

\section{Conclusion}

The opportunity to use the theorems known in pseudoanalytic functions theory was provided to us by the form of the equation (16). As we have shown, looking at $\varphi$ as the pseudoanalytic function of the first kind was useful since from this one can get important information regarding the solutions of our problem. For the future, we would like to mention that equation (12), which in terms of the function $u$, defined in (21), has the form of the Liouville equation, can also be related to one of the equations known in pseudoanalityc functions theory, called the Beltrami equation. It will be interesting for the future to also analyze it with this viewpoint in mind and possibly make novel conclusions.

Acknowledgement. This work was supported by the joint grant of Volkswagen Foundation and Shota Rustaveli National Science Foundation of Georgia (Ref. 93562 \& \# 04/48).

\section{References}

[1] G. Akhalaia, G. Giorgadze, G. Gulagashvili, The Analysis of Vortex Equations Using Methods of Generalized Analytic Functions,

Bull.TICMI, Vol.22, No.2, 2018, 135-141.

[2] G. Akhalaia, G. Giorgadze, V. Jikia, N. Kaldani, G. Makatsaria, N. Manjavidze, Elliptic Systems on Riemann Surfaces,

Lecture notes of TICMI, TSU press, Vol.13, 2012, 3-167. 
[3] L. Bers, An Outline Of Pseudoanalytic Functions, Vol.62, No.2, 1956, 291-331.

[4] A. Jaffe, C. Taubes, Vorteces and Monopoles, Birkhäuser Boston, 1980.

[5] I. Vekua, Generalized analytic functions, Pergamon, 1962. 